\title{
CONSTITUINTES QUÍMICOS E ATIVIDADE ANTIOXIDANTE DE EXTRATOS DAS FOLHAS DE Terminalia fagifolia Mart. et Zucc
}

\author{
Mariane Cruz Costa Ayres e Mariana H. Chaves* \\ Departamento de Química, Universidade Federal do Piauí, 64049-550 Teresina - PI, Brasil \\ Daniel Rinaldo, Wagner Vilegas e Gerardo Magela Vieira Júnior \\ Departamento de Química Orgânica, Instituto de Química, Universidade Estadual Paulista Júlio de Mesquita Filho, 14800-900 \\ Araraquara - SP, Brasil
}

Recebido em 5/8/08; aceito em 16/1/09; publicado na web em 3/7/09

\begin{abstract}
CHEMICAL CONSTITUENTS AND ANTIOXIDANT ACTIVITY FROM LEAVES EXTRACTS OF Terminalia fagifolia Mart. et Zucc. Phytochemical investigation of ethanolic leaves extracts of T. fagifolia led to the isolation of (+)-catechin, sitosterol-3-O$\beta$-D-glucopyranoside, $\alpha$ - and $\beta$-tocopherol, a mixture of lupeol, $\alpha$ - and $\beta$-amyrin, sitosterol and a mixture of glicosid flavonoids (CP-13). The structures of these compounds were identified by ${ }^{1} \mathrm{H}$ and ${ }^{13} \mathrm{C}$ NMR spectral analysis and comparison with literature data. Absolute configuration of the catechin was determinate by circular dichroism. Antioxidant activity $\left(\mathrm{EC}_{50}\right)$, evaluated by 2,2-diphenyl1-picrylhidrazyl (DPPH) assay system, decreased in the order: (+)-catechin $>$ hydroalcoholic fraction $>\mathrm{CP}-13>$ aqueous fraction $>$ EtOH extract.
\end{abstract}

Keywords: Terminalia fagifolia; DPPH, antioxidant activity.

\section{INTRODUÇÃO}

A família Combretaceae compreende 18 gêneros, sendo Combretum e Terminalia os mais abundantes, com cerca de 370 e 200 espécies, respectivamente, as quais são largamente distribuídas no Oeste e Sul da África e amplamente utilizadas na medicina popular. ${ }^{1}$

As plantas do gênero Terminalia são ricas em triterpenos pentacíclicos e seus derivados glicosilados, flavonoides, taninos e outros compostos aromáticos. ${ }^{2}$ Apresentam diversas atividades farmacológicas, tais como antifúngica, anti-helmíntica, antimalárica, anticancerígena, hipoglicêmica, anti-inflamatória, antibacteriana, antioxidante, antiulcerogênica, antiviral, antidepressora, tripanocida, moluscicida, imunomodulatória e efeitos hepatoprotetor, cardioprotetor, dentre outros. ${ }^{3}$

Terminalia fagifolia Mart. et Zucc é uma planta encontrada no cerrado, distribuída pela Bahia, Ceará, Goiás, Distrito Federal, Piauí, São Paulo, Maranhão, Mato Grosso, Mato Grosso do Sul e Minas Gerais. É conhecida popularmente como capitão, capitão-do-mato, capitão-docerrado, capitão-do-campo, mirindiba e pau-de-bicho. A árvore é melífera e ornamental, sendo seus frutos utilizados no artesanato e a madeira pode ser empregada na marcenaria e construção civil. Na medicina popular a casca do caule é usada no combate a aftas e tumores. ${ }^{4}$

Estudos realizados com T. fagifolia demonstraram que o extrato etanólico das cascas apresenta atividades citotóxica e antioxidante, bem como a ocorrência de flavonoides (flavanonas, chalconas e flavanas), 1,3-diarilpropanos, triterpenos pentacíclicos glicosilados e não-glicosilados e um esteroide, o sitosterol. ${ }^{5} \mathrm{O}$ extrato etanólico das folhas apresenta forte potencial antioxidante no ensaio do DPPH. ${ }^{6}$

Nos últimos anos, um grande interesse no estudo de antioxidantes tem ocorrido devido, principalmente, às descobertas sobre o efeito dos radicais livres no organismo. Os radicais livres são moléculas orgânicas ou inorgânicas e átomos, que contêm um ou mais elétrons não empareados, instáveis e muito reativos, que causam danos às células e patologias relacionadas como artrite, catarata, câncer, diabete, disfunção cerebral, aterosclerose, doenças cardíacas e neurodegenerativas, dentre outras. ${ }^{6-8}$

*e-mail: mariana@ufpi.br
A ação dos radicais livres pode ser bloqueada ou retardada por substâncias antioxidantes, as quais podem ser basicamente de duas categorias: naturais e sintéticas. Nesta última categoria estão incluídos o BHA (butil-hidroxi-anisol) e o BHT (butil-hidroxi-tolueno), comumente utilizados em alimentos contendo lipídios, porém apresentam problemas de segurança e toxicidade. Sendo assim, as pesquisas têm-se voltado no sentido de encontrar produtos naturais com potencial antioxidante que possam substituí-los ou ser usados em associação. ${ }^{6,7}$

Este artigo relata o estudo fitoquímico do extrato etanólico das folhas de T. fagifolia biomonitorado para a obtenção de substâncias antioxidantes.

\section{PARTE EXPERIMENTAL}

\section{Procedimentos experimentais gerais}

Os espectros de RMN ${ }^{1} \mathrm{He}$ e ${ }^{13} \mathrm{C}$ foram obtidos em espectrômetro Brüker modelo Avance DRX-500, operando a 300, $500 \mathrm{MHz}\left({ }^{1} \mathrm{H}\right)$, 75 e $125 \mathrm{MHz}\left({ }^{13} \mathrm{C}\right)$. As placas cromatográficas foram preparadas utilizando-se uma mistura de gel de sílica 60 G Vetec e 60 GF $_{254}$ Fluka (1:1) e as revelações das cromatoplacas foram feitas por borrifamento com solução de sulfato cérico. As colunas cromatográficas foram preparadas com gel de sílica 0,2-0,5 mm da Merck e 0,063-0,20 mm da Vetec ou Sephadex LH-20 da Aldrich.

Foram utilizados apenas solventes e reagentes analiticamente puros. O radical DPPH (2,2-difenil-1-picril-hidrazila) e o BHT (butilhidroxi-tolueno) foram adquiridos da Sigma Aldrich e a rutina da PVP SA., Parnaíba-PI. As medidas de absorção foram feitas usando-se espectrofotômetro UV-Vis Hitachi U-3000.

A análise de cromatografia líquida de alta eficiência acoplada a um detector de arranjo de fotodiodos e dicroísmo circular (CLAEDAD-DC) foi realizada em cromatógrafo da marca Jasco, bomba modelo PU-2089, acoplado a um detector de arranjo de fotodiodos (MD-2010) e um detector de dicroísmo circular (CD-2095) e injetor automático AS-2055. A coluna utilizada foi do tipo Chiralcel OD-H ( 250 x 4,6 mm, $5 \mu \mathrm{m})$, com fase móvel em hexano/EtOH (75:25 v/v) acidificados com $0,1 \%$ de TFA e vazão de $0,5 \mathrm{~mL} / \mathrm{min}$. 


\section{Material vegetal}

As folhas de T. fagifolia foram coletadas na cidade de Floriano - Piauí, Brasil, em setembro de 2002. A exsicata encontra-se depositada no Herbário Graziela Barroso da UFPI, com o número TEPB 18061.

\section{Extração e isolamento dos constituintes}

As folhas de T. fagifolia foram secas ao ar, moídas (505 g) e extraídas seis vezes por maceração com etanol à temperatura ambiente. O solvente foi removido em evaporador rotativo fornecendo $201 \mathrm{~g}$ de extrato etanólico. Parte deste extrato $(91 \mathrm{~g})$ foi suspenso em 900 $\mathrm{mL}$ de uma mistura de $\mathrm{H}_{2} \mathrm{O} / \mathrm{MeOH}(2: 1)$ e submetido à partição com acetato de etila, fornecendo as frações aquosa e acetato de etila. Esta última foi concentrada (37 g), suspensa em $\mathrm{MeOH} / \mathrm{H}_{2} \mathrm{O}(9: 1)$ e extraída com hexano, fornecendo as frações hidroalcoólica (29 g) e hexânica ( $3 \mathrm{~g})$. A fração hexânica concentrada foi dissolvida em metanol a quente (banho de água a $40^{\circ} \mathrm{C}$ ), mantida sob refrigeração por $24 \mathrm{~h}$ e filtrada, resultando em duas frações, o material solúvel em $\mathrm{MeOH}(2 \mathrm{~g})$ e o precipitado graxo $(0,9 \mathrm{~g})$.

A fração hidroalcoólica $(500 \mathrm{mg})$ foi dissolvida em $5 \mathrm{~mL}$ de $\mathrm{H}_{2} \mathrm{O}$ e aplicada em coluna Sep-Pak Waters Vac $35 \mathrm{cc}\left(\mathrm{C}_{18}, 10 \mathrm{~g}\right)$. Em seguida a coluna foi eluída com $\mathrm{H}_{2} \mathrm{O} / \mathrm{MeOH}$ em ordem decrescente de polaridade. ${ }^{9}$ A fração CP-4 (135 mg), eluída com $20 \%$ de $\mathrm{MeOH}$, forneceu, após filtração em Sephadex LH-20 com metanol, a substância (+)-catequina (1, $27 \mathrm{mg})$. A fração CP-6 (134 mg), eluída com $30 \%$ de $\mathrm{MeOH}$, correspondeu ao 3-O- $\beta$-D-glicopiranosídeo do sitosterol $(2,29 \mathrm{mg})$, que foi submetido à reação de acetilação com anidrido acético $(1 \mathrm{~mL})$ e piridina $(1 \mathrm{~mL})$, fornecendo o derivado tetra-acetilado (30 mg). A fração CP-13 (148 mg), eluída com 40\% de $\mathrm{MeOH}$, consistiu de uma mistura de flavonoides glicosilados de difícil separação.

O material solúvel em $\mathrm{MeOH}$ (2 g) foi fracionado por meio de cromatografia em coluna de gel de sílica (210 g), eluída com hexano, hexano/AcOEt e AcOEt/MeOH em ordem crescente de polaridade, fornecendo 104 frações (125 mL cada), sendo coletadas como segue: frações 1-8 (hexano 100\%), 9-24 (hexano/AcOEt, 98:2), 25-39 (hexano/AcOEt, 95:5), 40-52 (hexano/AcOEt, 9:1), 53-59 (hexano/AcOEt, 85:15), 60-70 (hexano/AcOEt, 7:3), 71-81 (hexano/AcOEt, 6:4), 82-87 (hexano/AcOEt, 1:1), 88-94 (AcOEt 100\%) e 95-104 (AcOEt/ $\mathrm{MeOH}, 1: 1)$. Após remoção do solvente em evaporador rotativo e análise por CCDA, as frações foram reunidas em 10 grupos.

Os grupos T1 (frações 20-24, 79 mg), T2 (frações 25-27, $139 \mathrm{mg}$ ) e T4 (frações 43-50, $108 \mathrm{mg}$ ) foram recromatografados em coluna de Sephadex LH-20 eluídas com hexano/ $\mathrm{CH}_{2} \mathrm{Cl}_{2}$ (1:4) fornecendo o $\alpha$-tocoferol (3, $24 \mathrm{mg}), \beta$-tocoferol $(\mathbf{4}, 8 \mathrm{mg})$ e sitosterol $(\mathbf{8}, 37 \mathrm{mg})$, respectivamente.

O grupo T3 (frações 34-36, $141 \mathrm{mg}$ ) foi suspenso em hexano originando $106 \mathrm{mg}$ de um sólido amorfo, que foi dissolvido em hexano/ $\mathrm{CH}_{2} \mathrm{Cl}_{2}$ (1:4) e submetido à cromatografia em coluna de Sephadex LH-20 com eluição isocrática neste solvente, fornecendo $18 \mathrm{mg}$ de uma mistura dos triterpenos $\alpha$-amirina (5), $\beta$-amirina (6) e lupeol (7).

(+)-Catequina (1): sólido amorfo alaranjado. $\mathrm{RMN}^{1} \mathrm{H}[300 \mathrm{MHz}$, $\left.\mathrm{CD}_{3} \mathrm{OD}, \delta(\mathrm{ppm})\right]: 4,59$ (d; $\left.J=7,4 \mathrm{~Hz} ; \mathrm{H}-2\right) ; 4,01$ (ddd, $J=8,0 ; 7,4$ e $5,4 \mathrm{~Hz} ; \mathrm{H}-3$ ); 2,52 (dd; $J=16,0$ e 8,0 Hz; H-4 ${ }_{\text {anti }}$ ); 2,84 (dd; $J=16,0$ e 5,4 Hz; H-4 ${ }_{\text {sin }}$ ); 5,96 (d; J=2,2 Hz; H-6); 5,89 (d; J=2,2 Hz; H-8); 6,85 (d, $J=1,8 \mathrm{~Hz} ; \mathrm{H}-2$ '); 6,79 (d; $J=8,1 \mathrm{~Hz} ; \mathrm{H}-5$ '); 6,72 (dd; $J=1,8$ e $8,1 \mathrm{~Hz}$; H-6'). RMN ${ }^{13} \mathrm{C}$ [75 MHz, $\mathrm{CD}_{3} \mathrm{OD}, \delta$ (ppm)]: 82,6 (C-2); 68,6 (C-3); 28,2 (C-4); 157,3 (C-5); 96,4 (C-6); 157,4 (C-7); 95,6 (C-8); 156,7 (C-9); 100,9 (C-10); 132,0 (C-1'); 115,2 (C-2'); 146,0 (C-3'); 146,0 (C-4'); 116,2 (C-5'); 120,1 (C-6').
$\alpha$-Tocoferol (3): $\mathrm{RMN}^{1} \mathrm{H}\left[500 \mathrm{MHz}, \mathrm{CDCl}_{3}, \delta\right.$ (ppm)]: 2,13 (s, $\left.6 \mathrm{H}, \mathrm{CH}_{3}\right) ; 2,17$ (s, 3H, $\left.\mathrm{CH}_{3}\right) ; 2,62(\mathrm{t}, J=6,7 \mathrm{~Hz}, 2 \mathrm{H}-4)$. RMN ${ }^{13} \mathrm{C}[125$ $\mathrm{MHz}, \mathrm{CDCl}_{3}, \delta$ (ppm)]: 74,5 (C-2); 31,6 (C-3), 20,8 (C-4); 118,5 (C5); 144,5 (C-6); 121,0 (C-7); 122,6 (C-8); 23,8 (C-2a); 117,4 (C-4a); 11,3* (C-5a); 12,2* (C-7a); 145,5 (C-8a); 11,8* (C-8b); 39,8 (C-1'); 21,0 (C-2'); 37,5 (C-3', 5', 7', 9'); 32,8 (C-4', 8'); 24,4 (C-6’); 24,8 (C-10'); 39,4 (C-11'); 28,0 (C-12'); 22,7 (C-13', 12'a); 19,7 (C-4'a, 8 'a). *Os valores podem ser permutados.

$\beta$-Tocoferol (4): RMN ${ }^{1} \mathrm{H}\left[500 \mathrm{MHz}, \mathrm{CDCl}_{3}, \delta\right.$ (ppm)]: 2,09 (s, 3H, $\left.\mathrm{CH}_{3}\right) ; 2,11$ (s, 3H, $\mathrm{CH}_{3}$ ); 6,49 (s, 1H-7), 2,61 (t, J=6,9 Hz, $2 \mathrm{H}-4)$. RMN ${ }^{13} \mathrm{C}\left[125 \mathrm{MHz}, \mathrm{CDCl}_{3}, \delta\right.$ (ppm)]: 74,5 (C-2); 31,4 (C3), 20,8 (C-4); 119,1 (C-5); 145,7 (C-6); 115,3 (C-7); 124,1 (C-8); 23,8 (C-2a); 120,3 (C-4a); 11,0 (C-5a); 146,0 (C-8a); 15,8 (C-8b); 39,7 (C-1'); 21,0 (C-2'); 37,4 (C-3', 5', 7', 9'); 32,8 (C-4', 8'); 24,4 (C-6'); 24,8 (C-10'); 39,4 (C-11'); 28,0 (C-12'); 22,7 (C-13', 12'a); 19,6 (C-4'a, 8'a).

\section{Análise quantitativa da atividade antioxidante}

A investigação da atividade antioxidante do extrato EtOH, frações hidroalcoólica e aquosa, CP-13 e (+)-catequina (1) foi realizada quantitativamente pelo método do DPPH, utilizando como controle positivo a rutina e o BHT. O método consistiu no monitoramento do consumo do radical livre $\mathrm{DPPH}^{\bullet}$ pelas amostras, em soluções de concentrações 25, 50, 100, 150, 200 e $250 \mu \mathrm{g} / \mathrm{mL}$, através da medida do decréscimo da absorbância. ${ }^{6}$ Estas medidas foram feitas em espectrofotômetro UV-Vis, no comprimento de onda de $516 \mathrm{~nm}$, sendo que a curva de calibração utilizada para se obter a concentração de DPPH no meio após a reação com o extrato foi $[\mathrm{DPPH}]=35,846 X-0,230$, onde $X$ é absorbância e $\mathrm{r}=0,9997$. As leituras das medidas de absorbância das amostras $(0,3 \mathrm{~mL}$ da solução metanólica da amostra ou do controle positivo e $2,7 \mathrm{~mL}$ da solução estoque de DPPH em metanol na concentração de $40 \mu \mathrm{g} /$ $\mathrm{mL}$ ) foram realizadas no primeiro, quinto e décimo minuto e em seguida a cada $10 \mathrm{~min}$ até completar $1 \mathrm{~h}$ de experimento. A mistura de metanol $(2,7 \mathrm{~mL})$ e solução metanólica do extrato $(0,3 \mathrm{~mL})$ foi utilizada como branco. Com os dados obtidos foi determinada a porcentagem de atividade antioxidante $\left\{\% \mathrm{AA}=100-\left[\mathrm{Abs}_{\text {amostra }}-\right.\right.$ $\left.\left.\left.\mathrm{Abs}_{\text {branco }}\right) \mathrm{x} 100\right] / \mathrm{Abs}_{D P P H}\right\}$, onde $\mathrm{Abs}_{D P P H}$ é a absorbância inicial da solução metanólica de DPPH e Abs amostra é a absorbância da mistura reacional (DPPH+amostra). ${ }^{6}$

A concentração eficiente - $\mathrm{CE}_{50}$ - capaz de reduzir o DPPH em $50 \%$, no tempo de 30 min de reação, foi obtida através de uma exponencial de primeira ordem, plotando-se as concentrações de cada amostra versus porcentagem de DPPH remanescente $\left(\% \mathrm{DPPH}_{\mathrm{REM}}\right){ }^{6}$ usando o programa Microcal Origin 7.5. Todas as análises foram realizadas em triplicata. Os valores da média de três repetições $(n=3) \pm$ desvio padrão da média foram calculados utilizando o programa Excel.

\section{RESULTADOS E DISCUSSÃO}

\section{Identificação dos constituintes químicos isolados}

O fracionamento do extrato etanólico das folhas de T. fagifolia conduziu ao isolamento e identificação estrutural de um flavonoide (1), dois tocoferóis (3 e 4), três triterpenos em mistura (5-7) e dois esteroides ( 2 e 8) (Figura 1). O $\beta$-tocoferol (4) é inédito no gênero Terminalia e, com exceção do sitosterol $(\mathbf{8}),{ }^{5}$ todas as demais substâncias são inéditas em T. fagifolia. A identificação estrutural foi baseada na análise dos espectros de $\mathrm{RMN}{ }^{1} \mathrm{H}$ e ${ }^{13} \mathrm{C}$ e comparação com dados da literatura, sendo a configuração absoluta da catequina determinada por dicroísmo circular. 

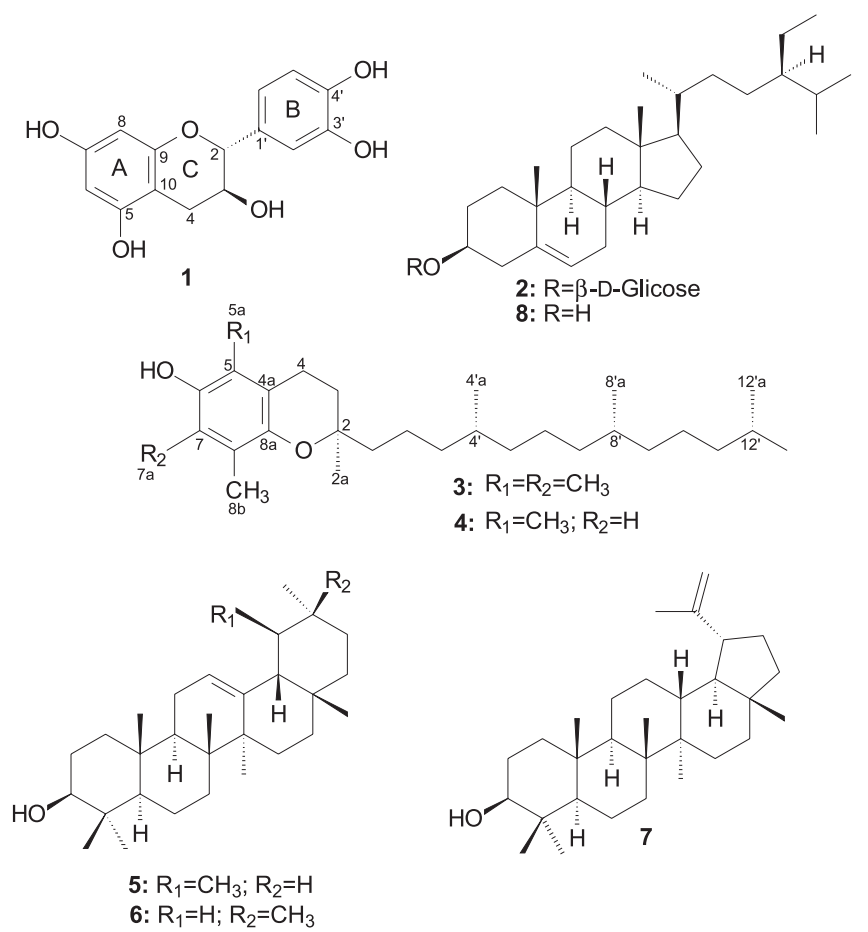

Figura 1. Estruturas das substâncias isoladas do extrato etanólico das folhas de T. fagifolia

As substâncias 1, 2 e a fração CP-13 foram isoladas da fase hidroalcoólica. A identificação estrutural de $\mathbf{1}$ como catequina foi realizada por meio da análise dos espectros de $\mathrm{RMN}{ }^{1} \mathrm{H}$ e ${ }^{13} \mathrm{C}$, mostrando-se consistentes com os relatados por Galotta e colaboradores. ${ }^{10}$

Catequinas podem existir como dois isômeros trans-catequina e cis-epicatequina, dependendo da estereoquímica configuracional dos grupos hidroxila e 3',4'-diidroxifenil ligados a C-3 e C-2 do anel C. Cada um dos diasteriosômeros existe como dois isômeros ópticos: $(2 \mathrm{R}$, 3S)-2,3-trans-(+)-catequina e (2S, 3R)-2,3-trans-(-)-catequina, (2R, 3R)-2,3-cis-(+)-epicatequina e (2S, 3S)-2,3-cis-(-)-epicatequina. ${ }^{11}$

Embora os estereoisômeros (+)-catequina e (-)-epicatequina sejam os mais comumente encontrados em plantas, a substância $\mathbf{1}$ foi submetida à análise por cromatografia líquida de alta eficiência acoplada a um detector de arranjo de fotodiodos e dicroísmo circular (CLAE-DAD-DC), em coluna quiral, para elucidação da estereoquímica absoluta. O cromatograma obtido apresentou apenas um pico, indicando a presença de um único isômero. A curva de dicroísmo circular correspondente (Figura 2) mostrou dois efeitos Cottons negativos a 280 e $220-240 \mathrm{~nm}$, definindo as configurações R e S para C-2 e C-3, respectivamente. ${ }^{12,13}$ Este resultado, associado à análise dos espectros de RMN de 1, e a comparação destes dados com aqueles de um padrão autêntico indicaram que se trata da (2R, 3S)-2,3-trans(+)-catequina. Este é o segundo relato desta substância no gênero Terminalia, tendo sido isolada anteriormente de $T$. chebula ${ }^{14}$ enquanto a (-)-catequina foi relatada somente em T. argentea. ${ }^{15}$

A fração CP-13 mostrou-se constituída por uma mistura complexa de flavonoides glicosilados de difícil separação, os quais foram evidenciados no espectro de $\mathrm{RMN}{ }^{1} \mathrm{H}$, pelos sinais característicos de hidrogênios em anel aromático $(\delta 6,05-8,06)$, e em carbonos oximetínicos de açúcares $(\delta 3,35-5,20){ }^{16}$

A substância 2, após tratamento com anidrido acético e piridina, foi identificada como o derivado tetra-acetilado do 3-O- $\beta$-Dglicopiranosídeo do sitosterol. Os dados de $\mathrm{RMN}$ de ${ }^{1} \mathrm{H} \mathrm{e}{ }^{13} \mathrm{C}$ foram consistentes com aqueles relatados na literatura para a estrutura proposta, ${ }^{17}$ sendo adicionalmente observados no espectro de RMN
${ }^{1} \mathrm{H}$ sinais entre $\delta$ 2,02-2,19 relativos aos hidrogênios metílicos dos grupos acetatos, e no espectro de $\mathrm{RMN}{ }^{13} \mathrm{C}$, os sinais entre $\delta 20,5$ 20,7 e $\delta 169,3-170,6$ atribuídos aos carbonos metílicos e carbonílicos, respectivamente.

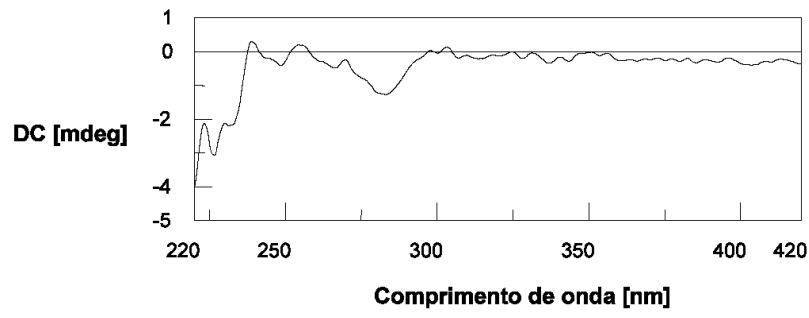

Figura 2. Curva de dicroísmo circular (DC) da substância 1

O fracionamento do material solúvel em $\mathrm{MeOH}$, proveniente da fração hexânica da partição do extrato $\mathrm{EtOH}$, resultou no isolamento e identificação de dois tocoferóis $(\alpha$ e $\beta),{ }^{18}$ uma mistura de triterpenoides pentacíclicos (lupeol, $\alpha$ - e $\beta$-amirina) ${ }^{19}$ e o sitosterol. ${ }^{20}$ Estas substâncias são comumente relatadas em outras espécies vegetais, inclusive da família Combretaceae, sendo identificadas por comparação com dados da literatura. Para identificação da mistura de triterpenos foi utilizada a metodologia de Olea e Roque. ${ }^{21}$

\section{Atividade antioxidante}

O extrato etanólico da T. fagifolia apresentou atividade antioxidante (AA) avaliada pelo ensaio do DPPH, conforme relatado anteriormente. ${ }^{6}$

Após partição do extrato etanólico, a atividade antioxidante foi maior na fração hidroalcoólica, evidenciada pelos valores de concentração eficiente capazes de reduzir o DPPH em 50\% $\left(\mathrm{CE}_{50}\right)$, conforme apresentado na Figura 3. Os valores da $\mathrm{CE}_{50}$ obedecem a seguinte ordem: fração hidroalcoólica < fração aquosa < extrato EtOH.

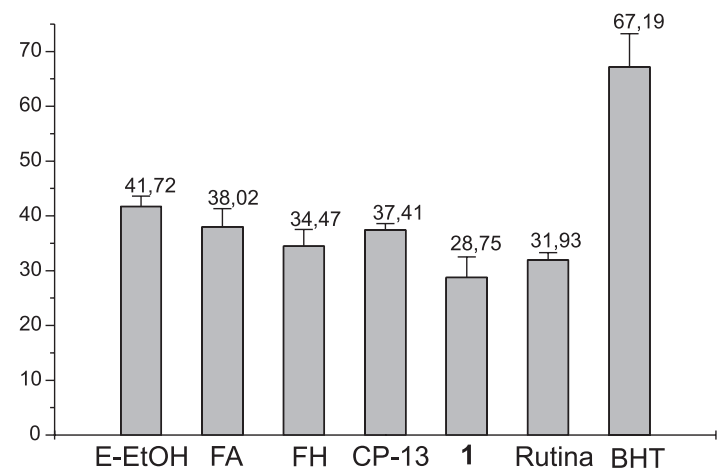

Figura 3. Concentração eficiente $\left(C E_{50}, e m \mu \mathrm{g} / \mathrm{mL}\right)$ do extrato etanólico (E$\mathrm{EtOH})$ e frações da T. fagifolia. FA: fração aquosa; FH: fração hidroalcoólica; CP-13: mistura de flavonoides glicosilados; 1: (+)-catequina

$\mathrm{A} \mathrm{CE}_{50} \mathrm{em} \mu \mathrm{g} / \mathrm{mL} \mathrm{da}(+)$-catequina $(28,75 \pm 3,78)$ foi menor que a da CP-13 $(37,41 \pm 1,18)$ e dos controles positivos BHT $(67,19 \pm$ $6,04)$ e rutina $(31,93 \pm 0,52)$. No entanto, considerando os valores de $\mathrm{CE}_{50}$ em $\mu \mathrm{mol} / \mathrm{mL}$, verificou-se que a (+)-catequina $\left(9,90 \pm 1,30 \times 10^{-2}\right)$ tem menor atividade antioxidante que o controle positivo rutina $(5,20$ $\left.\pm 0,09 \times 10^{-2}\right)$, devido às massas moleculares serem bem diferentes, 290 e $600 \mathrm{~g} / \mathrm{mol}$, respectivamente. Os resultados obtidos mostraram que o alto potencial antioxidante da fase hidroalcoólica e do extrato EtOH da T. fagifolia foi decorrente, pelo menos em parte, da presença da (+)-catequina e da fração CP-13. 
A porcentagem de atividade antioxidante (\%AA) das amostras de T. fagifolia e dos controles positivos (rutina e BHT), nas concentrações de $25,50,100,150,200$ e $250 \mu \mathrm{g} / \mathrm{mL}$, está apresentada na Figura 4. Foi verificado que a \%AA é dependente da concentração, porém acima de $150 \mu \mathrm{g} / \mathrm{mL}$ não ocorre variação significativa, exceto para a (+)-catequina, fração hidroalcoólica e rutina (controle positivo), as quais não apresentaram variação a partir de $100 \mu \mathrm{g} / \mathrm{mL}$. O extrato EtOH e frações da T. fagifolia exibiram atividade antioxidante superior ao BHT em todas as concentrações testadas. A (+)-catequina apresentou \%AA superior à fração CP-13, nas concentrações de 25 a $100 \mu \mathrm{g} / \mathrm{mL}$, sendo a única das amostras analisadas a atingir o valor de aproximadamente $50 \%$ a $25 \mu \mathrm{g} / \mathrm{mL}$; no entanto, a $50 \mu \mathrm{g} / \mathrm{mL}$ a rutina foi superior à (+)-catequina.

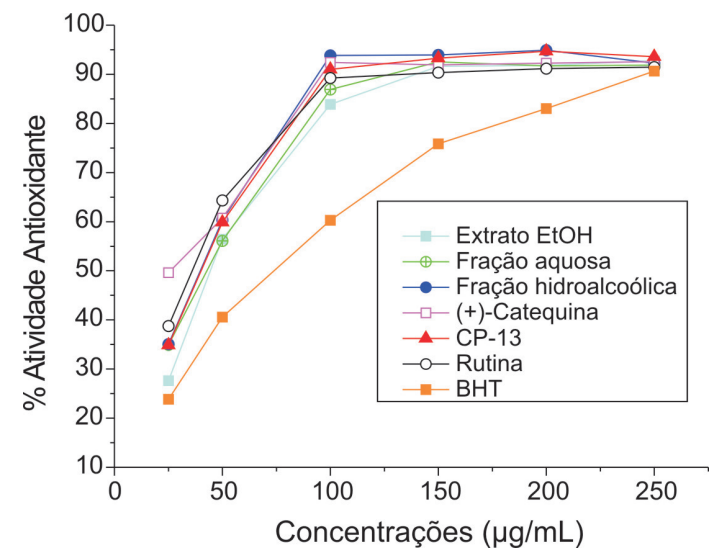

Figura 4. Porcentagem de atividade antioxidante em função das concentrações no tempo de 30 min de reação

Os flavonoides possuem diferentes atividades antioxidantes decorrentes da habilidade para sequestrarem espécies reativas de oxigênio e nitrogênio, as quais estão diretamente relacionadas a alguns critérios estruturais tais como o número de hidroxilas fenólicas, a presença do sistema orto-diidroxi no anel B, uma ligação dupla $\mathrm{C}_{2}-\mathrm{C}_{3}$ conjugada com a função 4-oxo no anel $\mathrm{C}$ ou de um grupo hidroxila no $\mathrm{C}-3 .{ }^{22-24}$

$\mathrm{A}(+)$-catequina (1) não apresenta em sua estrutura a ligação dupla em $\mathrm{C}_{2}-\mathrm{C}_{3}$ conjugada com a função 4-oxo no anel $\mathrm{C}$, justificando assim sua menor atividade antioxidante, avaliada pela $\mathrm{CE}_{50}$, em relação à rutina. Contudo, seu excelente desempenho como antioxidante natural é justificado não só pelos demais critérios estruturais citados anteriormente, mas também por apresentar, diferentemente de outros flavonoides como a rutina, um hidrogênio benzílico em relação ao anel B, que poderá ser facilmente abstraído por radicais livres. ${ }^{23}$

As propriedades das catequinas têm sido amplamente reportadas na literatura, mostrando que são responsáveis pelos efeitos benéficos de muitos alimentos e bebidas, como chocolates, chás, frutos e vinhos. Catequina e epicatequina apresentam atividade sequestradora do radical peroxila dez vezes maior que o L-ascorbato (vitamina $\mathrm{C}$ ) e $\beta$-caroteno. ${ }^{25}$ Portanto, todas estas informações justificam a excelente atividade antioxidante encontrada no extrato etanólico e fração hidro- alcoólica da T. fagifolia, uma vez que a principal substância isolada e responsável por esta atividade é a (+)-catequina (1).

\section{AGRADECIMENTOS}

Ao CNPq, CAPES e FINEP pela bolsa concedida a M. H. Chaves e pelo apoio financeiro. Ao CENAUREMN pelos espectros. À Dra. G. M. Sousa do Herbário Graziela Barroso, UFPI, pela identificação do material botânico.

\section{REFERÊNCIAS}

1. Masoko, P.; Picard, J.; Eloff, J. N.; S. Afr. J. Bot. 2007, 73, 173.

2. Araújo, D. S.; Chaves, M. H.; Quim. Nova 2005, 6, 996; Garcez, F. R.; Garcez, W. S.; Miguel, D. L. S.; Serea, A. A. T.; Prado, F. C.; J. Braz. Chem. Soc. 2003, 14, 461.

3. Araújo, D. S.; Dissertação de Mestrado, Universidade Federal do Piauí, Brasil, 2005; Dwivedi, S.; J. Ethnopharmacol. 2007, 114, 114; Manna, P.; Sinhá, M.; Sil, P. C.; Pathophysiology 2007, 14, 71.

4. Almeida, S. P.; Proença, C. E. B.; Sano, S. M.; Ribeiro, J. F.; Cerrado: espécies vegetais úteis, EMBRAPA-CPAC: Planaltina, Distrito Federal, 1998.

5. Garcez, F. R.; Garcez, W. S.; Santana, A. L. B. D.; Alves, M. M.; Matos, M. F. C.; Scaliante, A. M.; J. Braz. Chem. Soc. 2006, 17, 1223.

6. Sousa, C. M. M.; Silva, H. R.; Vieira Jr., G. M.; Ayres, M. C. C.; Costa, C. L. S.; Araújo, D. S.; Cavalcante, L. C. D.; Barros, E. D. S.; Araújo, P. B. M.; Brandão, M. S.; Chaves, M. H.; Quim. Nova 2007, 30, 351.

7. Barreiros, A. L. B. S.; David, J. M.; David, J. P.; Quim. Nova 2006, 29, 113; Bianchi, M. L. P.; Antunes, L. M. G.; Rev. Nutr. 1999, 12, 123; Valko, M.; Izakovic, M.; Mazur, M.; Rhodes, C. J.; Telser, J.; Mol. Cell. Biochem. 2004, 266, 37.

8. Kumaran, A.; Karunakaran, R. J.; Food Chem. 2006, 97, 109.

9. Materska, M.; Perucka, I.; J. Agric. Food Chem. 2005, 53, 1750.

10. Galotta, A. L.; Boaventura, M. A.; Lima, L. A. R. S.; Quim. Nova 2008 , $6,1427$.

11. Jung, M. J.; Heo, S.-I.; Wang, M.-H.; Food Chem. 2008, 108, 482.

12. Slade, D.; Ferreira, D.; Marais, J. P.J.; Phytochemistry 2005, 66, 2177.

13. Korver, O.; Wilkins, C.; Tetrahedron 1971, 27, 5459.

14. Kashiwad, Y.; J. Nat. Prod. 1992, 55, 1033.

15. Garcez, F. R.; Garcez, W. S.; Martins, M.; Lopes, F. A.; Biochem. Syst. Ecol. 2003, 31, 229.

16. Pizzolatti, M. G.; Cunha Jr., A.; Szpoganicz, B.; Sousa, E.; Braz-Filho, R.; Schripsema, J.; Quim. Nova 2003, 26, 466.

17. Fang, J.-M.; Wang, K.-C.; Cheng, Y.-S.; Phytochemistry 1991, 30, 3383.

18. Matsuo, M.; Urano, S.; Tetrahedron 1976, 32, 229.

19. Mahato, S. B.; Nandy, A. K.; Roy, G.; Phytochemistry 1992, 31, 2199.

20. De-Eknamkul, W.; Potduang, B.; Phytochemistry 2003, 62, 389.

21. Olea, R. S. G., Roque, N. F.; Quim. Nova 1990, 13, 278.

22. Burda, S.; Oleszek, W.; J. Agric. Food Chem. 2001, 49, 2774.

23. Kondo, K.; Kurihara, M.; Miyata, N.; Suzuki, T.; Toyoda, M.; Arch. Biochem. Biophys. 1999, 362, 79.

24. Vieira Júnior, G. M.; Sousa, C. M. M.; Cavalheiro, A. J.; Lago, J. H. G.; Chaves, M. H.; Helv. Chim. Acta 2008, 91, 2159.

25. Yilmaz, Y.; Trends Food Sci. Technol. 2006, 17, 64. 